MODELING, IDENTIFICATION AND CONTROL, 1991, VOL. 12, NO. 2, $81-94$

doi:10.4173/mic.1991.23

\title{
Nonlinear decoupling in process control
}

\author{
JENS G. BALCHEN†
}

Keywords: Nonlinearity, decoupling, inverse dynamics, process control.

The principle of nonlinear decoupling as a general approach to the control of nonlinear processes is reviewed. A novel technique is introduced for resolving the inverse problem in case the nonlinear process function does not have a closed-form inverse. The paper also indicates the relationships between nonlinear decoupling, 'cascade control' and 'sliding mode control'.

\section{Review}

The principle of nonlinear decoupling has been suggested by many authors for decades (Balchen 1963) and a number of authors in recent years have described related ideas sometimes under different names (Freund 1975, Isidori et al. 1981, Balchen et al. 1987, Lie and Balchen 1988). In robotics literature, an almost similar principle is known under the name of 'inverse dynamics' or 'computed torque technique' (Bejczy 1974, Luh et al. 1980).

A multivariable process is described by a nonlinear state space model of the form

$$
\begin{aligned}
\dot{\boldsymbol{x}} & =\boldsymbol{f}(\boldsymbol{x}, \boldsymbol{u}, \boldsymbol{v}) \\
\boldsymbol{z} & =\boldsymbol{g}(\boldsymbol{x})
\end{aligned}
$$

where

$\boldsymbol{x}$ state vector, $\operatorname{dim} \boldsymbol{x}=\mathrm{n}$

$u$ control vector, $\operatorname{dim} u=\mathrm{r}$

$v$ disturbance vector, $\operatorname{dim} v=s$

$z$ property vector, $\operatorname{dim} z=m \leqslant r$

$f$ vector of nonlinear functions

$g$ vector of nonlinear functions

'Nonlinear decoupling' has the objective of finding the control vector $u$ which alters the process in such a way that the property vector $z$ changes with a specified desired rate $\dot{z}_{d}$.

According to (1) and (2) we have

$$
\dot{z}=\frac{\partial g(x)}{\partial x} \dot{x}=\frac{\partial g(x)}{\partial x}-f(x, u, v)
$$

(3) is to be solved with respect to $u$ when $\dot{z}=\dot{z}_{d}$ and in general we will get

$$
\boldsymbol{u}=\boldsymbol{h}\left(\boldsymbol{x}, \boldsymbol{v}, \dot{z}_{d}\right)
$$

Received 22 March 1991.

$\uparrow$ Division of Engineering Cybernetics, The Norwegian Institute of Technology, University of Trondheim, 7034 Trondheim, Norway. 
Most often the $\boldsymbol{h}(\cdot)$ will not be available in closed form so that an implicit solution becomes necessary through some iterative procedure. However, in some special cases, a solution of the form (4) may be found. Such is the case when (1) and (2) take the form

$$
\begin{aligned}
& \dot{x}=f(x, v)+B(x) u \\
& z=D x
\end{aligned}
$$

where $B(\cdot)$ and $D$ are matrices of appropriate dimensions. Since (5) is linear in $u$ we find the solution equivalent to (4) as

$$
u=(D B(x))^{-1}\left(\dot{z}_{d}-D f(x, v)\right)
$$

The matrix $D B(\cdot)$ must be nonsingular for the nonlinear decoupling solution or (7) to exist. This presents certain structural constraints on the process and the way the property vector is related to the state vector.

When nonlinear decoupling is possible according to (4) or (7), then the nonlinear process has been replaced by a set of $m$ independent integrators as seen from $\dot{z}_{d}$ to $z$. These integrators may be controlled by a diagonal control matrix with constant elements as shown in Fig. 1 in which it has been chosen that

$$
\dot{z}_{d}=G\left(z_{0}-z\right)
$$

where $z_{0}$ is the setpoint of the property vector.

By choosing large values of the elements of $\mathrm{G}$, the time constants of the individual first order control loops will be small. In fact, the matrix $\mathbf{G}$ is the eigenvalue matrix of the resulting system.

\section{Iterative inverse}

As the inverse equation of (4) will not usually be available in closed form, some kind of equation solver must be used. One way of doing this is to introduce a new differential equation to solve (3) with respect to $u$.

$$
\dot{u}=K(\cdot)\left(\dot{z}_{d}-\frac{\partial g(x)}{\partial x} f(x, u, v)\right)
$$

where $K(\cdot)$ is a matrix which has the purpose of securing the convergence of (9).
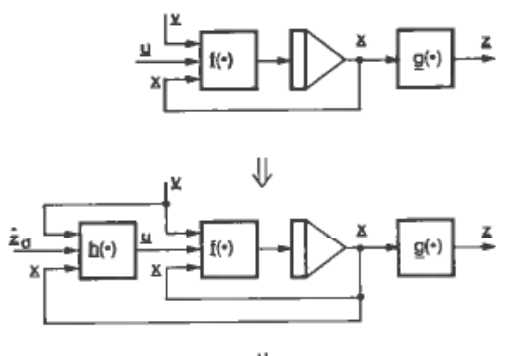

$\Downarrow$

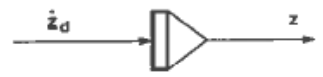

$\Downarrow$

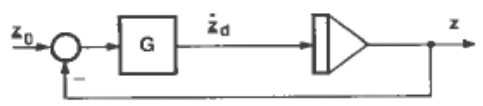

Figure 1. Block diagram representation of elementary nonlinear decoupling. 


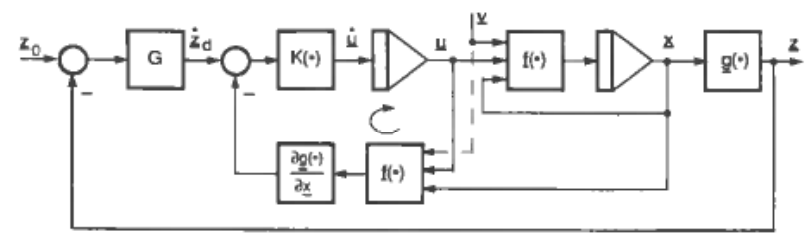

Figure 2. Nonlinear decoupling and multivariable control employing 'iterative inverse'.

In the steady state $(\dot{\boldsymbol{u}}=0)$, the right-hand side of (9) will be identical to (3).

A block diagram illustrating the complete control system with nonlinear decoupling is shown in Fig. 2. Here the solution of (4) has been replaced by (9). Note that (4) and (9) will only be identical when $K$ of (9) has such large values that $\dot{u}$ becomes very small. The dynamics of the new auxiliary loop will now be of importance to the system behaviour.

The block diagram in Fig. 2 clearly reveals some very important features of the new solution. It is seen that the function

$$
\frac{\partial \boldsymbol{g}(\cdot)}{\partial \boldsymbol{x}} \boldsymbol{f}(\cdot)
$$

appears in the feedback of the auxiliary loop. This indicates the inversion of the process model. Furthermore it is seen that if the disturbance (v) or at least some of its elements are known, then we have a precise indication of what to do with these variables to achieve nonlinear decoupling. In other words, we have not only solved the decoupling problem, but also the feedforward control problem. The disturbance elements that are not measurable should be replaced by estimated values, the most primitive of which would be the expected mean values.

Another observation from Fig. 2 is that the solution requires the full state vector $x$ to be available for the computations. This may be totally realistic in a case when all states are measured. Note that the property vector $z$ is not necessarily the same as the measurement vector $\boldsymbol{y}$, even though in some cases it may be. In case not all state variables $(\boldsymbol{x})$ are measured, one could replace $\boldsymbol{x}$ by an estimated state vector $\hat{\boldsymbol{x}}$ derived from a special state estimator (e.g. state observer or Kalmar filter). We shall not address this problem, but assume that the state variables are available.

The matrix $K(\cdot)$ must be determined so as to achieve acceptable convergence of the auxiliary loop. This may be done in a number of ways. The simplest is to assume that the loop has small perturbations $\delta \boldsymbol{u}$ around a nominal value of $\boldsymbol{u}$. Thus we get the differential equation for the perturbations of the auxiliary loop

$$
\delta \dot{u}=-K(\cdot) \frac{\partial g(\cdot)}{\partial \boldsymbol{x}} \frac{\partial f(\cdot)}{\partial \boldsymbol{u}} \delta \boldsymbol{u}
$$

If we specify that (10) is to have prescribed eigenvalues defined by the eigenvalue matrix $\Lambda$, such that

$$
\delta \dot{u}=\Lambda \delta \boldsymbol{u}
$$

we get

$$
K(\cdot)=-\Lambda\left(\frac{\partial g(\cdot)}{\partial \boldsymbol{x}} \frac{\partial f(\cdot)}{\partial \boldsymbol{u}}\right)^{-1}
$$




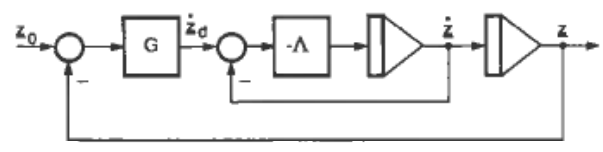

Figure 3. The dynamic effect of nonlinear decoupling employing 'iterative inverse'.

The eigenvalues $\Lambda$ should be specified to be much larger than the eigenvalues of the uncontrolled process so that the dynamics of the auxiliary loop will not influence the total control too much. When they are large, the block diagram of Fig. 2 may be reduced to that shown in Fig. 3 which illustrates that the decoupling has been achieved, but with a slight dynamic action ahead of the integration process.

The matrix $G$ may now be chosen to give an acceptable response from the outer loop in Fig. 3. It is easily seen that $G=-\Lambda$ or $G=-\frac{1}{2} \Lambda$ will give satisfactory results.

\section{Nonlinear decoupling applied to cascade control}

As has already been pointed out, the matrix DB of (7) must have an inverse in order that nonlinear decoupling can exist. The equivalent requirement is that

$$
\frac{\partial g(\cdot)}{\partial x} \frac{\partial f(\cdot)}{\partial u}
$$

of (12) is to have an inverse. The choice of the property vector $z$ is not at all obvious. In fact, in many cases the structure of the mathematical model of (1) is such that it is impossible to conceive of a property vector which makes elementary nonlinear decoupling feasible. Such is the case when the process consists of a number of cascaded dynamic elements, the end output of which represents the most reasonable property description. In such a case the elementary nonlinear decoupling structure will not exist.

If the elementary conditions for an inverse to exist are not satisfied, it may still be possible to arrange the system so that an 'indirect' control of the property vector can be realized using the principle of nonlinear decoupling.

Consider the system

$$
\begin{aligned}
& \dot{x}_{1}=f_{1}\left(x_{1}, u\right) \\
& \dot{x}_{2}=f_{2}\left(x_{1}, x_{2}\right) \\
& \dot{x}_{3}=f_{3}\left(x_{2}, x_{3}\right)
\end{aligned}
$$

Here $x_{1}$, with $\operatorname{dim} x_{1}=\operatorname{dim} u=r$, is the substate which has 'the strongest connection' to the control vector $u$. Then $x_{2}$, having $\operatorname{dim} x_{2}=r$, is an intermediate state which can be regarded as having $x_{1}$ as its controls. Furthermore $x_{3}$, having $\operatorname{dim} x_{3}=r$, can be regarded as being controlled by $x_{2}$ and is the one related to the property vector so that

$$
z=g\left(x_{3}\right)
$$

Now nonlinear decoupling can be applied sequentially as follows:

1. Determination of the control vector based upon

$$
\begin{aligned}
\dot{u} & =K_{1}(\cdot)\left(\dot{x}_{1 d}-f_{1}(\cdot)\right) \\
\dot{x}_{1 d} & =G_{1}\left(x_{10}-x_{1}\right)
\end{aligned}
$$


2. The active control of $(14)$ will be via $\left(x_{10}\right)$ such that

$$
\begin{aligned}
& \dot{x}_{10}=K_{2}(\cdot)\left(\dot{x}_{2 d}-f_{2}(\cdot)\right) \\
& \dot{x}_{2 d}=G_{2}\left(x_{20}-x_{2}\right)
\end{aligned}
$$

3. And finally the indirect control via $x_{2}$ of the property vector through (15) by

$$
\begin{aligned}
& \dot{x}_{20}=K_{3}(\cdot)\left(\dot{z}_{d}-\frac{\partial g(\cdot)}{\partial x_{2}} f_{3}(\cdot)\right) \\
& \dot{z}_{d}=G_{3}\left(z_{0}-g\left(x_{3}\right)\right)
\end{aligned}
$$

A block diagram of this solution is shown in Fig. 4 where it is clearly seen that we have a typical structure of cascade control or inner loops. These loops are different however, from those known in elementary linear control in that they are nonlinear loops.

Obviously the bandwidths of these loops become progressively lower going outwards, but since the innermost loops usually can be made very fast, the outer loops will still have acceptable bandwidths. However, since the new dynamics has been introduced, the nonlinear decoupling will no long be ideal.

Another approach to this type of problem which has been studied by a number of authors (Freund 1975, Isidori 1986, Kravaris and Soroush 1988, Sastry and Isidori 1989) leads to a type of derivative feedback from the property vector which yields an effective linearization of the system.

An example used by Kravaris and Soroush (1988) to illustrate that particular method, is used here to illustrate nonlinear decoupling applied to cascade control.

\section{Example}

The system under consideration is (Kravaris and Soroush 1988, Lie 1990)

$$
\begin{aligned}
& \dot{x}_{1}=-x_{3}+x_{1} u_{1} \\
& \dot{x}_{2}=x_{1}^{2}-2 x_{1} u_{1} \\
& \dot{x}_{3}=-x_{3} x_{1}+x_{4}+\left(1+x_{1}\right) x_{1} u_{1} \\
& \dot{x}_{4}=u_{2}
\end{aligned}
$$

The property vector to be controlled is

$$
z=\left[\begin{array}{l}
z_{1} \\
z_{2}
\end{array}\right]=\left[\begin{array}{l}
x_{1} \\
x_{2}
\end{array}\right]
$$

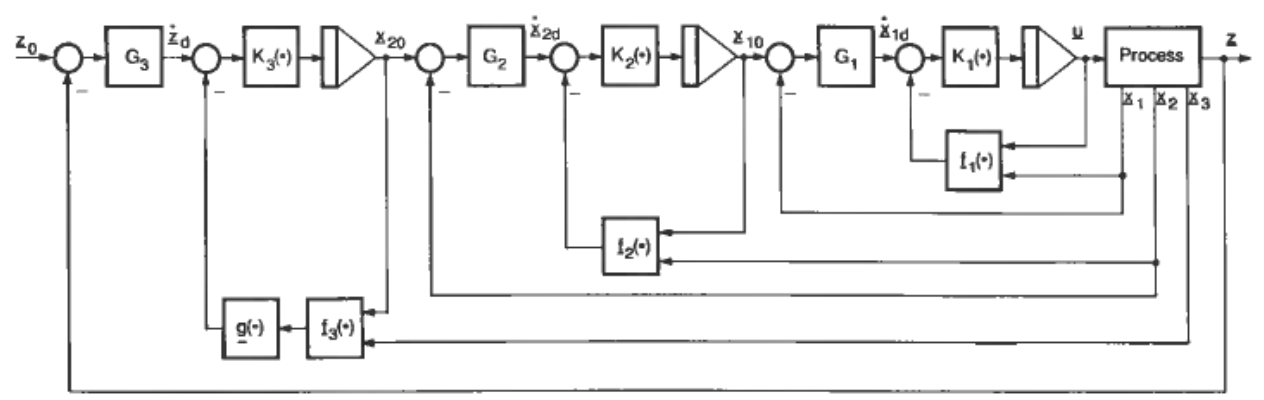

Figure 4. Nonlinear decoupling applied to cascade control. 
It is immediately clear that the matrix

$$
\frac{\partial g(\cdot)}{\partial x} \cdot \frac{\partial f(\cdot)}{\partial u}=\left[\begin{array}{llll}
1 & 0 & 0 & 0 \\
0 & 1 & 0 & 0
\end{array}\right]\left[\begin{array}{cc}
x_{1} & 0 \\
-2 x_{1} & 0 \\
\left(1+x_{1}\right) x_{1} & 0 \\
0 & 1
\end{array}\right]=\left[\begin{array}{cc}
x_{1} & 0 \\
-2 x_{1} & 0
\end{array}\right]
$$

is singular. This means that the elementary nonlinear decoupling does not exist. Observing (22) (26) it is obvious that the state $x_{4}$ can be controlled at infinite bandwidth through a strong feedback to $u_{2}$. Thereby $x_{4}$ can be regarded as a new control input replacing $u_{2}$. Furthermore the state $x_{3}$ may be controlled through a nonlinear decoupling with $x_{4}$ as the input. A strong feedback around $\dot{x}_{3}$ will then eliminate the dynamics of (24) with the result that $x_{3}$ may be regarded as the control input to (22). Thereby (22) and (23) are the only equations left and elementary nonlinear decoupling may be applied to these.

In this example the state variable $x_{4}$ plays the role of the state vector $x_{1}$ in (16) above, the state variable $x_{3}$ plays the role of $x_{2}$ in (18) and as given by (26), the first two state variables represent the property vector equivalent to $x_{3}$ in (21).

A block diagram showing the final solution is given in Fig. 5. Here loop I governs $x_{4}$, loop II governs $x_{3}$, loop III governs $x_{2}$ and loop IV governs $x_{1}$.

\section{Limitations on the control vector-avoiding integrator wind-up}

When applying the 'iterative inverse' technique discussed above, particular attention should be paid to the problem of limitations on the control vector. In most physical processes there will be definite limitations on the magnitude that can be achieved in each of the control variables. This is especially so when a control variable is

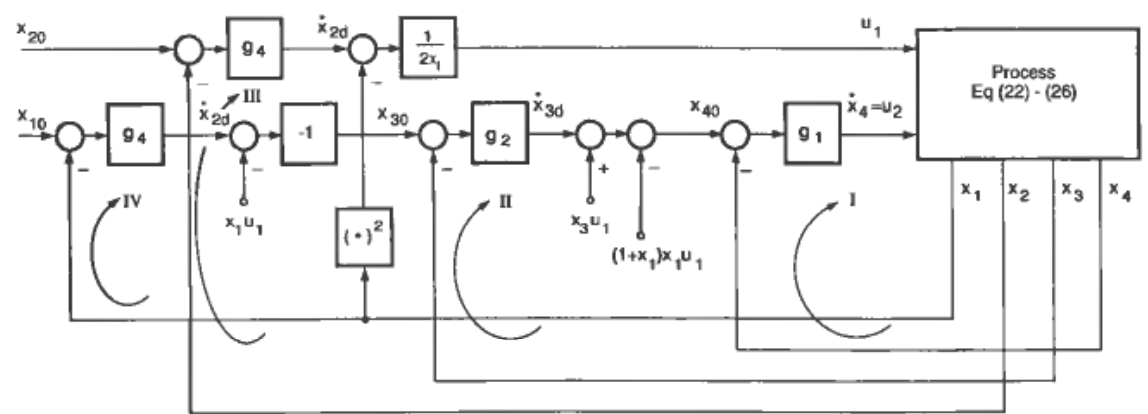

Figure 5. Nonlinear decoupling applied to system in Eqns. (22)-(26).

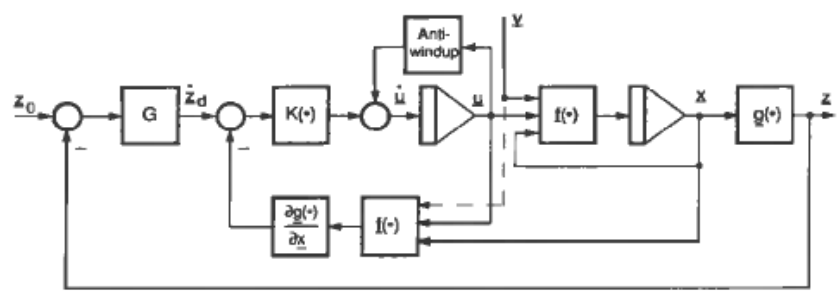

Figure 6. Anti wind up-algorithm applied to system with saturation in control vector. 
the flow through a valve or the torque produced by a motor. In the 'iterative inverse' approach the control vector is generated by a set of integrators, which will be subject to 'wind up' if no precautions are taken. The solution to this problem is shown in Fig. 6 which is the same as Fig. 2, except for the 'anti-wind up' element that has been added. This element acts as a feedback around all the integrators ensuring that $\boldsymbol{u}$ does not exceed its limit values. The effect of this mechanism is very significant as will be seen in the example below.

\section{Example}

Assume a scalar process model to be

$$
\begin{aligned}
& \dot{x}=-x+(1+a x) u \\
& z=x
\end{aligned}
$$

Employing the iterative inverse, we get

$$
K=-\lambda\left(\frac{\partial f}{\partial u}\right)^{-1}=\frac{-\lambda}{1+a x}
$$

The resulting system has the structure of Fig. 2 and is now studied under different conditions in Fig. 7. A unit step is applied to $z_{0}=x_{0}$ when $g=50, \lambda=-100, a=1$. Figures $7(a)$ and $(b)$ illustrate the resulting control actions $u(t)$ and the state $x(t)$ with and without limitation on the control $\left(u_{\max }=5\right)$. As is clearly seen, the limitation of the control gives a significant wind-up action which is definitely detrimental. Similar curves are shown in Figs. $8(a)$ and $8(b)$ for the case when $u_{\max }=2 \cdot 5$. This shows an even more severe influence of the control limitation compared to the case without any limitation. Figures $9(a)$ and $9(b)$ show the same case as in Fig. 7, but with anti-wind-up
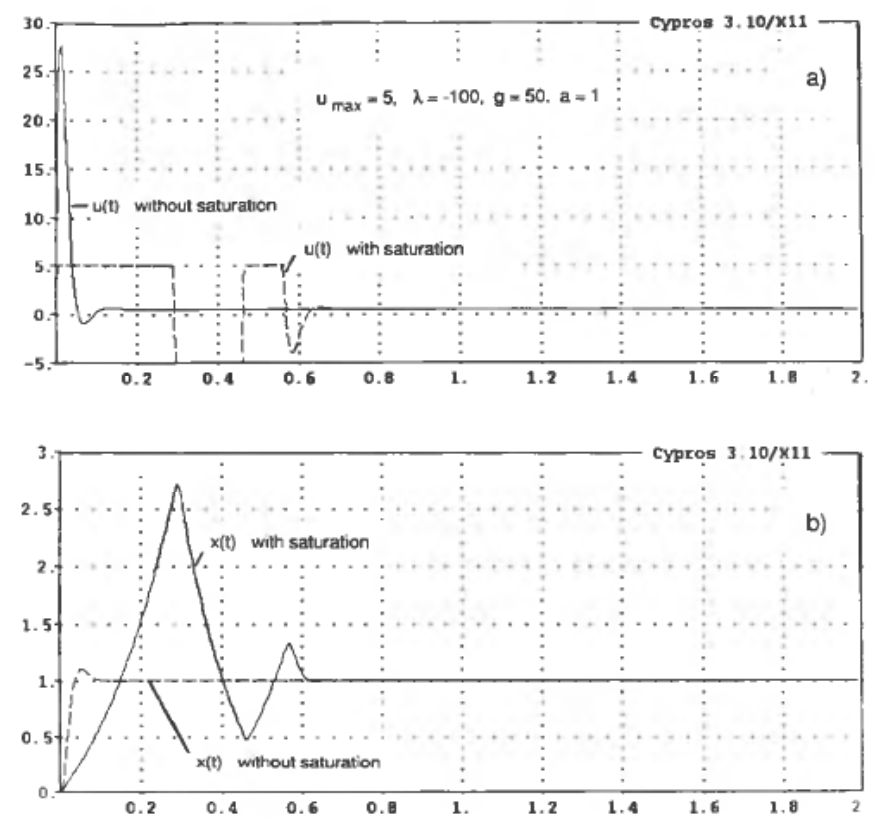

Figure 7. Time response of system in Eqns. (28)-(29) with system structure as in Fig. 2 following a step in $z_{0}$ (control saturation $u_{\max }=5 \cdot 0$ ). 

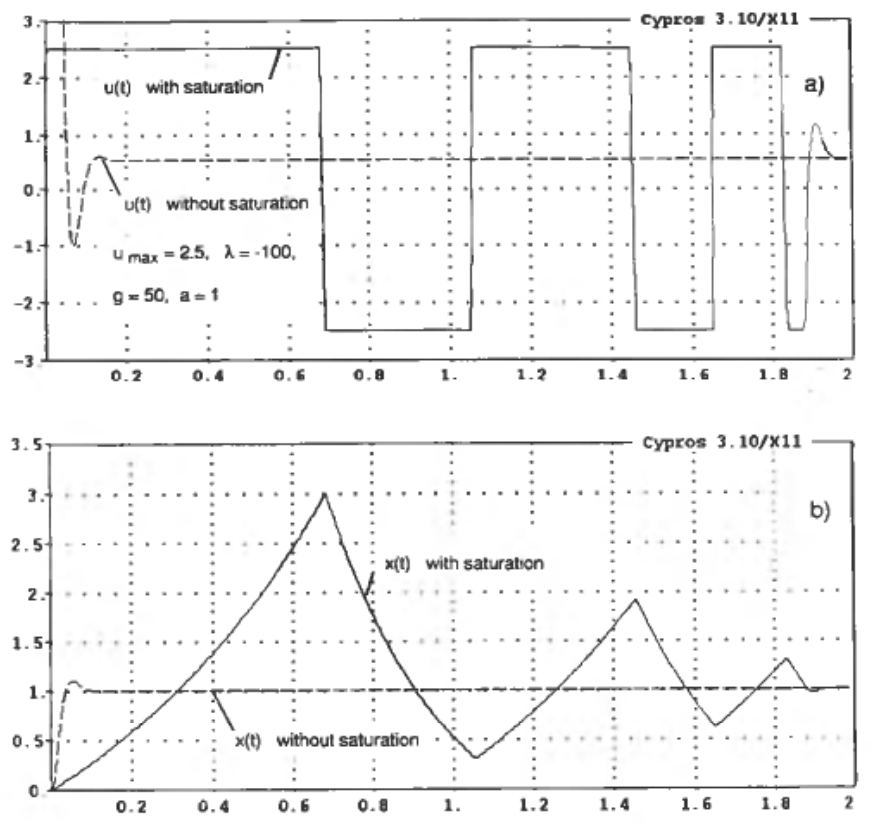

Figure 8. Same as Fig. 7 but with control saturation $u_{\max }=2 \cdot 5$.
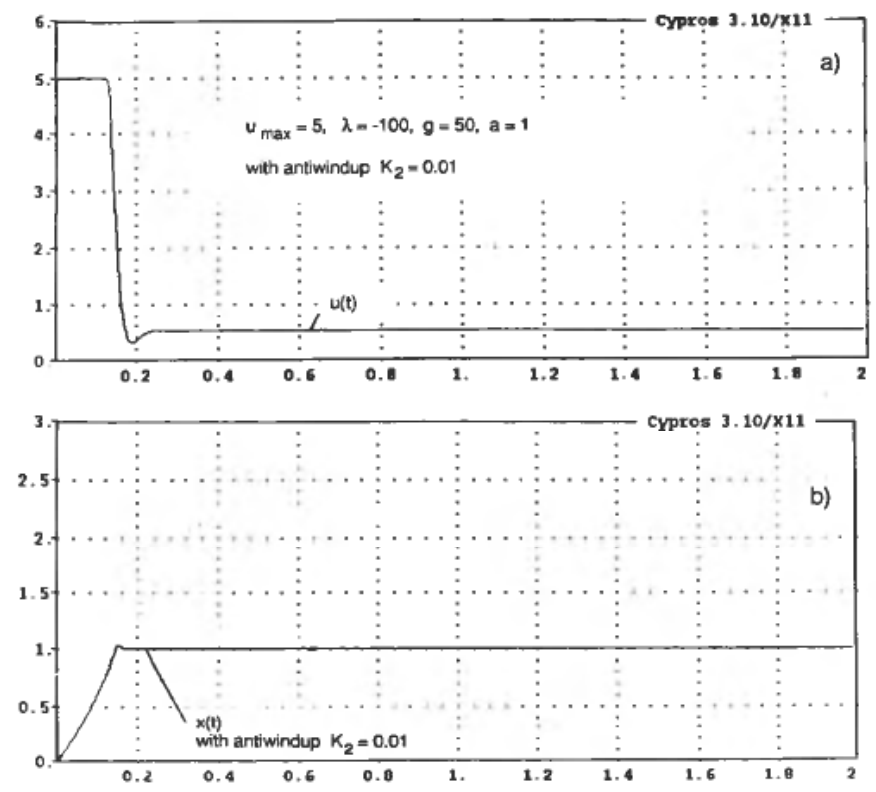

Figure 9. Same problem is in Fig. 7 but with system structure of Fig. 6 with anti wind upalgorithm. 
feedback around the integrator in the auxiliary loop (the gain of the anti-wind-up loop is $\left.K_{2}=0,01\right)$. Here it is clear that the detrimental effect of the control limitations is totally removed, but the response speed of the state is lower than in the unlimited case as is to be expected.

\section{Nonlinear decoupling in processes with non-minimum-phase behaviour}

A multivariable nonlinear process may exhibit physical phenomena which show non-minimum-phase behaviour. As is well known, the inversion of such a dynamic phenomenon may lead to the necessity of a predictive compensator. For example, the inverse of a transportation lag is a predictor. But even though non-minimum-phase elements may appear in a multivariable process description, it does not necessarily means that the inverse dynamics does not exist. The only requirement for the inverse to exist, is that matrix $D B$ of (7) is nonsingular. The non-minimum-phase behaviour of the process will appear in matrix $B$ and in some cases make matrix $D B$ singular or $(D B)^{-1}$ ill conditioned. An example of a complex dynamic process which exhibits nonminimum-phase behaviour, is the Fluid Catalytic Cracker (FCC), but in this case the $D B$ matrix is non-singular and thus a nonlinear decoupling can be realized for this process.

\section{The robustness of nonlinear decoupling}

The basic solution of decoupling as described in Fig. 2 assumes that the mathematical model as given in (1) is known. This is obviously an ideal requirement which will only be satisfied with a certain degree of accuracy in a real case. The function $g(\cdot)$ of $(2)$ is something chosen by the control designer and may therefore be assumed to be perfectly accurate. The uncertainty applies only to the structure and the parameters of the function $f(\cdot)$.

If for simplicity we assume that $z=x$, requiring that $\operatorname{dim} u=\operatorname{dim} x$ we get the simplest case.

The uncertainty of the model can be related to its parameters and/or to its structure. The latter most frequently means that the number of state variables in the real process is different (usually larger) than the number of state variables in the model. In our first attempt to analyze the robustness problem, we shall assume that the model structure is correct, that is the number of state variables of the system is the same as that of the model. Furthermore, in order to simplify the problem we shall assume that the process is linear so that

$$
\dot{\boldsymbol{x}}=A_{p} \boldsymbol{x}+\boldsymbol{B}_{p} \boldsymbol{u}
$$

where $A_{p}$ and $B_{p}$ refer to the real process.

Now we have

$$
\begin{aligned}
& A_{p}=\left(I+E_{A}\right) A_{m} \\
& B_{p}=\left(I+E_{B}\right) B_{m}
\end{aligned}
$$

where $A_{m}$ and $B_{m}$ refer to the model implemented in the control strategy and $E_{A}, E_{B}$ are respective uncertainty matrices. 
We then get

$$
\begin{aligned}
\dot{x} & =\left(I+E_{A}\right) A_{m} x+\left(I+E_{B}\right) B_{m} B_{m}^{-1}\left(G\left(x_{0}-x\right)-A_{m} x\right) \\
& =\left(I+E_{B}\right) G\left(x_{0}-x\right)+\left(E_{A}-E_{B}\right) A_{m} x \\
& \approx\left(I+E_{B}\right) G\left(x_{0}-x\right)
\end{aligned}
$$

where the feedback matrix $\mathrm{G}$ has large elements compared to those of the matrix $\left(E_{A}-E_{B}\right) A_{m}$. Apparently this system can tolerate fairly large uncertainties $E_{A}$ and $E_{B}$ before the system behaviour becomes much different from the ideal behaviour. This result is mainly due to the fact that the model structure was assumed to be correct. It confirms the well-known result that strong feedback around a process makes the total system insensitive to process parameter variations.

It follows from (33) that the uncertainty matrix $E_{B}$ will become larger when the matrix $B_{p}$ is close to being singular. This is seen by inverting (33) which leads to

$$
B_{p}^{-1}=B_{m}^{-1}\left(I+E_{B}\right)^{-1} \approx B_{m}^{-1}\left(I-E_{B}\right)
$$

and

$$
\frac{1}{\left|B_{p}\right|} \operatorname{adj} B_{p} \approx \frac{1}{\left|B_{m}\right|} \operatorname{adj} B_{m} \cdot\left(I-E_{B}\right)
$$

which yields

$$
E_{B} \approx I-\frac{\left|B_{m}\right|}{\left|B_{p}\right|} \operatorname{adj} B_{p}\left(\operatorname{adj} B_{m}\right)^{-1}
$$

If $B_{p}$ is close to singularity, then $\left|B_{p}\right| \rightarrow 0$ whereas $\left|B_{m}\right|$ remains larger. That indicates that $E_{B}$ will attain large values since $\left|B_{p}\right|$ appears in the denominator of (37). The lesson to be learned from this is that nonlinear decoupling is bound to be less robust when $B$ is close to singularity. This is not surprising because it means that the inverse model is illconditioned. This again tells us that since the $B$ matrix is a result of the way that the control variables have been chosen, we should take care and try to physically arrange our control variables so that the $B$ matrix does not have an ill-conditioned inverse. Another way to express this is that the eigenvalues of matrix $B$ are to be of the same order of magnitude.

Another approach to the question of robustness of nonlinear decoupling is demonstrated in Lie and Balchen (1990).

Comparing the requirement of $(D B)$ being nonsingular with the conditions for controllability we conclude that the first is most rigorous, i.e. even though a system is controllable it may not lend itself to decoupling.

\section{The problem with unmeasured states}

In the previous developments it has been assumed that the total state vector $\boldsymbol{x}$ is fully available in the computations. This may not be realistic in some cases, therefore this problem needs some attention.

Usually the solution to this problem in process control is to apply a state estimator and in some cases this may be feasible. The state vector then needed in the calculation, will be obtained from the state estimator as shown in Fig. 10 rather than from the process. Here $\boldsymbol{y}$ is the vector of measurements from the real process and $\hat{\boldsymbol{y}}$ are those estimated by the state estimator utilizing a model of the process. Here an 'Extended 


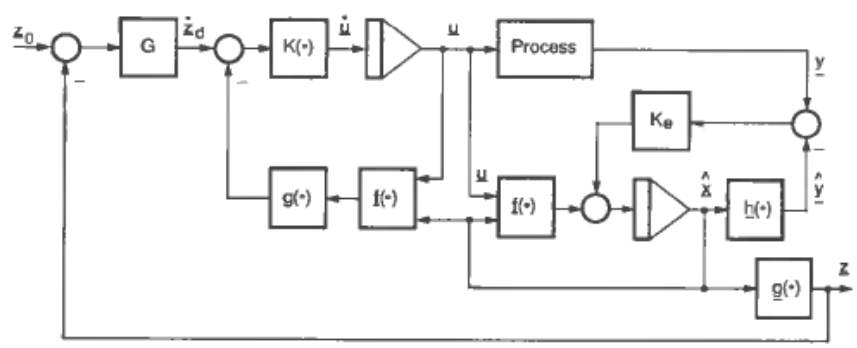

Figure 10. Employing a full state estimator in nonlinear decoupling.

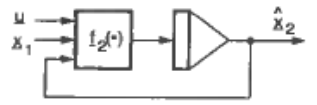

Figure 11. Direct calculation of a nonmeasurable states by means of submodel without corrective feedback.

Kalman Filter' structure is employed. The solution is somewhat contradictory, however, because the 'Extended Kalman Filter' assumes an internal feedback which involves the same problem as the one we are trying to solve by means of nonlinear decoupling. In practice, however, this problem may not be so severe because many of the states of the process are actually directly measurable and therefore do not have to be estimated. Thus only the unmeasured states are to be included in the estimator. In some cases the estimator for the unmeasured states may even be approximated by the model for these states driven by the control variables and the measured states without any corrective feedback from the output measurements as shown in Fig. 11.

\section{Nonlinear decoupling in batch control}

Batch processes have attained a new popularity in recent years because of their simplicity and the possibility for small scale production of sophisticated products. In the control of batch processes, it is often important to individually control the different states of the process in order to arrive at prescribed values of these states at given times, for example. Nonlinear decoupling will be a very powerful tool to achieve such goals. This is particularly so because nonlinear decoupling has resulted in as many first order processes (integrators) as the number of state variables in the process and the batch control of each of these processes is a very simple matter. However, since it is likely that the control variables will reach their saturation values, it is important to apply the 'antiwind-up' facility described above.

\section{Combining nonlinear decoupling with other control strategies}

The main idea with nonlinear decoupling is to remove severe effects of nonlinearities and interactions in the processes. This idea may be advantageous to any kind of control strategy.

The simplest control strategy is probably that of multiple monovariable PIDcontrollers applied to a multivariable process. This simple solution may work perfectly well when the process is not too nonlinear and the interactions are not detrimental to 
that particular control method. But in other cases it will be difficult to make it produce acceptable performance. If nonlinear decoupling of the process is applied before the multiple PI-controller (diagonal controller) this may give an excellent solution. As can be seen from the previous discussion, this only means that the control matrix $\mathrm{G}$, which is a diagonal proportional controller, is simply replaced by a diagonal PI-controller (i.e. each of the elements have an integrating element in parallel). This will not change the 'high frequency' behaviour of the system, rather improve the 'low frequency' accuracy of the control.

A similar argument can be made with regard to adaptive control. It is frequently suggested that adaptive control could be applied to processes involving major nonlinear phenomena in order to compensate for large changes in gains and dynamics. In some cases this may turn out to be possible, in others impossible. When the physical mechanisms of the process are known so that mathematical modeling is feasible, a nonlinear decoupling could be applied to the process in order to remove the largest contributions to nonlinear behaviour and interactions. The result will be a process with less nonlinear behaviour and interactions which could well be controlled by a set of adaptive controllers (or even constant controllers as described above). Figure 12 illustrates these arguments. The choice of method is in fact related to how much

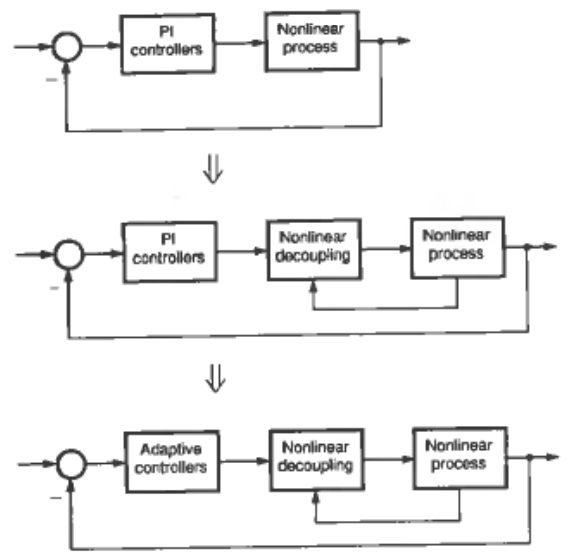

Figure 12. Nonlinear decoupling in relation to conventional control and adaptive control.
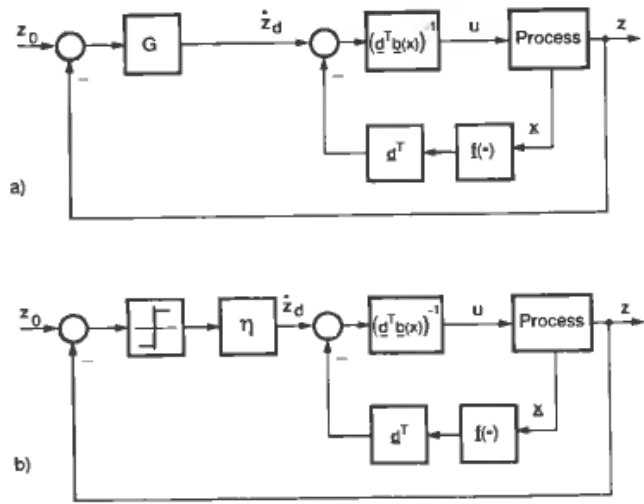

Figure 13. The similarity between (a) non linear decoupling and $(b)$ 'sliding mode control'. 
knowledge is available about the process. This again has to do with the qualifications of the control system designer. There is a general tendency towards the opinion that better process understanding, i.e. process modeling, is the best way to arrive at better control systems.

Finally, the relationships between nonlinear decoupling and the so-called 'sliding mode-control' will be indicated (Utkin 1977, Slotine and Li 1991).

Consider the system described by (5) and (6), where $u=u$ and $z=z$ are scalars. The nonlinear decoupling solution for this system is shown in Fig. 13(a) whereas the 'sliding mode control' solution is shown in Fig. 13(b).

What has been termed property vector (variable) above is called 'sliding surface' in the sliding mode literature.

The behaviour of the two solutions is quite similar when gain G in Fig. 13(a) is increased towards infinity and limitation (antiwind-up element) is imposed on the control variable $u$. Note that since the decoupling system from $\dot{z}_{d}$ to $z$ in the ideal case is an integrator there will be no stability problem in either solution.

\section{Conclusions}

The principles of nonlinear decoupling or inverse dynamics control have been reviewed and some significant properties of this powerful technique have been illustrated. Particular attention has been paid to the 'iterative inverse' which is a very direct way of applying the process model to the determination of the control actions that perform nonlinear decoupling. Also a method of 'multi step' nonlinear decoupling has been introduced for cases when the dimension of the control vector is significantly smaller than the dimension of the state. Finally, the applications of combining nonlinear decoupling with other control strategies, e.g. adaptive control, and the relationship to 'sliding mode control' have been pointed out.

\section{ACKNOWLEDGMENT}

The author acknowledges the assistance of Mr. Nils Martin Gørbitz in performing some simulation.

\section{REFERENCES}

BalChen, J. G. (1963). Control Engineering Vol. 1. (pp. 203), (Tapir Book Company, Trondheim), (in Nowegian).

Balchen, J. G., Lie, B., and SolBerg, I. (1987). Internal Decoupling in Nonlinear Process Control. Proc. IF AC 10th World Congress, Munich, 1987, edited by R. Isermann, Vol. 3, pp. 134139, (Pergamon Press) also published in Modeling, Identification and Control, 1988, 9, pp. 137-148.

BejCZy, A. K. (1974). Robot Arm Dynamics and Control. JPL Technical Memo, 33-369.

FreUnd, E. (1975). The structure of Decoupled Nonlinear Systems, Int. J. Contr., 21, 651-654.

Isidori, A., Krener, A. J., Gori Giorgi, C. and Monaco, S. (1981). Nonlinear Decoupling via Feedback: A Differential Geometric Approach". IEEE Trans. Automat. Control, 26, $331-345$.

Isidori, A., Krener, A. J., Gori Giorgi, C. and Monaco, S. (1981). Nonlinear Decoupling via Feedback: A Differential Geometric Approach. IEEE Trans. Automat. Control, 26, $331-345$.

Kravaris, C. and Soroush, M. (1988). Multivariable Nonlinear Control by Global Input/Output Linearization. Paper No. 129a, American Institute of Chemical Engineers, 1988 Annual Meeting, Washington DC. 
94

Nonlinear decoupling in process control

Lie, B. and Batches, J. G. (1988). Nonlinear Decoupling Applied to a Polypropene Reactor. Paper no. 129-E, American Institute of Chemical Engineers, 1988, Annual Meeting, Washington DC.

LIE, B. and BALChEN, J. G. (1990). Robustness of Nonlinear Decoupling in a Polypropene Reactor. In: Rijnsdorp, J. E. et al. (editors): Proceedings DYCORD+'89, Maastricht, The Netherlands, August 21-23, 1989 (Pergamon Press, Oxford).

LIE, B. (1990). Control Structures for Polymerization Processes Applied to Polypropene Manufacturing. Dr.ing. thesis, Division of Engineering Cybernetics, The Norwegian Institute of Technology, Trondheim, Norway.

Luth, J. Y. S., WALKer, M. W. and Paul, R. C. (1980). Resolved Acceleration Control of Mechanical Manipulators. IEEE Trans. on Automat. Control, 25, 468-474.

SASTRY, S. S. and Isidor, A. (1989). Adaptive Control of Linearizable Systems. IEEE Trans. on Automat. Control, 34, 1123-1131.

Slotine, J.-J. E. and Li, W. (1991). Applied Nonlinear Control (Prentice-Hall International Inc.). UTKIN, V. I. (1977). Variable structure systems with sliding modes: A survey. IEEE Trans. Automat. Control, 22, pp. 212-222. 